\title{
Collagen Turnover in Regenerating Barbels of a Catfish
}

\author{
Mary WhITEAR \\ Department of Biology, University College London, United Kingdom \\ Received January 31, 1990
}

\begin{abstract}
Summary. In regenerating barbels of Heteropneustes fossilis, membrane-enclosed collagen fibrils, some within lysosomes, are found in fibroblasts, in old connective tissue at the wound site and also in the fibroblasts of the newly-formed core of the regenerate. The implication is that tissue remodelling can involve fibroblasts in phagocytosis of recently formed collagen fibrils. Cytological evidence suggests that individual fibroblasts are capable of synthesis and phagocytosis simultaneously.
\end{abstract}

Although it has been known for 30 years that remodelling of connective tissue involves both synthesis and degradation of collagen, there is still not complete agreement about the intimate activities of the fibroblasts concerned. At present, it is a controversial issue whether collagen fibrils are formed in deep pits in fibroblasts (TRELSTAD and HAYASHI, 1979) or if such profiles represent phagocytosed fibrils (MARCHI and LEBLOND, 1983). The vast literature on the subject has mainly concerned mammalian or embryonic tissues, often in tissue culture. In the regenerating barbels of a catfish, newly-formed tissue can be distinguished from that existing before wounding, by position and by microscopical characters. Catfish barbels have bundles of collagen fibrils aligned longitudinally between the axial skeleton and the epidermis (BAECKER, 1926) and regenerate readily if truncated. The situation provides a useful model for further investigation.

\section{MATERIAL AND METHODS}

Heteropneustes fossilis is an air-breathing Indian catfish with eight long barbels. Specimens were maintained in aquarium tanks at $25-26^{\circ} \mathrm{C}$. Two individuals, standard lengths 80 and $85 \mathrm{~mm}$, were used. The distal parts of two barbels at a time were snipped off, at intervals of a day or more, with the fish anaesthetized either by cold or with MS 222 . When the fishes were killed, by an overdose of MS 222 , and the barbels fixed for electron microscopy, individual barbels had regenerated for periods ranging from two to eight days. Fixation was in a mixture of $1 \%$ osmium tetroxide (2 parts) and $2.5 \%$ glutaraldehyde (1 part) cacodylate-buffered to $\mathrm{pH}$ 7.4. Subsequent preparation for transmission electron microscopy involved dehydration in graded ethanol and embedding in araldite. Sections were stained with uranyl acetate and lead citrate and viewed in a Jeol JEM-100CXII instrument.

\section{RESULTS}

Two days after operation the barbel stump was covered with epidermis, at three days a dome of regenerated tissue was present. After five days, 1-1.5 $\mathrm{mm}$ of regenerated barbel had grown out, extending to between 2.4 and $3.5 \mathrm{~mm}$ after seven or eight days. The rate of growth was not quite consistent but was approximately $0.5 \mathrm{~mm}$ per day after healing of the wound. The regenerate was more slender and more flexible than the normal barbel.

In the connective tissue surrounding the skeletal rod of the Heteropneustes barbel the collagen fibrils are aligned parallel with the long axis of the barbel and show considerable variation in diameter. In Figure 1 a tract of such fibrils, diameters $30-120 \mathrm{~nm}$, is cut transversely at the level of the wound site, three days after truncation of the barbel. The location was determined by the presence of the stump of the skeleton together with regenerating nerve masses and some extravasated erythrocytes. Leucocytic macrophages, sometimes with large lysosomal bodies, were present, mainly in the epidermis but a few in the core of the barbel. Fibroblasts of the connective tissue were recognized by their character- 

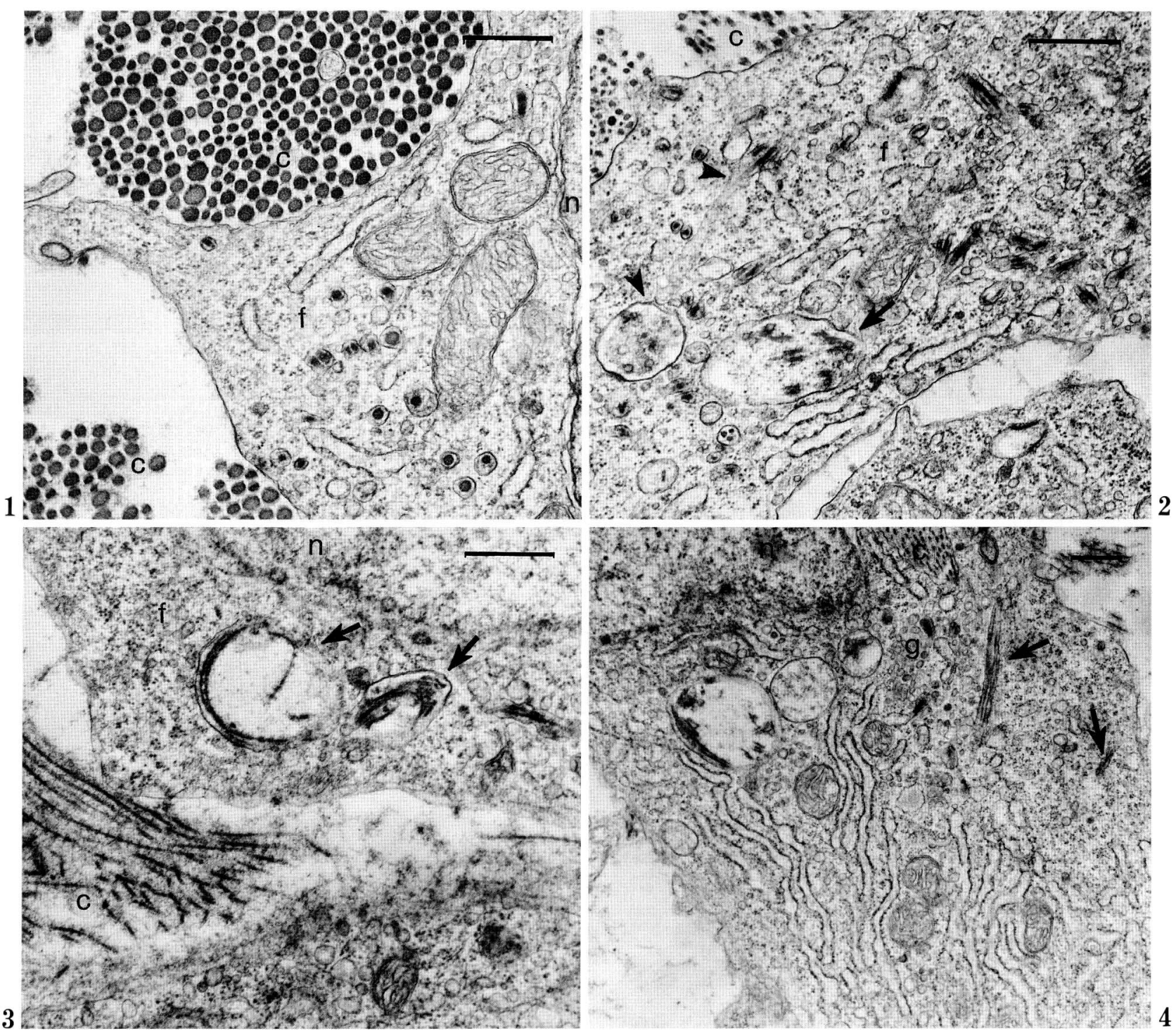

Fig 1. Transverse section of a barbel at the level of the wound site, 3 days after operation, showing collagen fibrils enclosed within a fibroblast. Scale-bar: $0.5 \mu \mathrm{m}$

Fig 2. Similar to Figure 1. The fibroblast contains a lysosomal profile (arrow) containing vesicles and fibrils. Between the two arrowheads, in the thickness of the section, membrane extends from a pair of enclosed fibrils to the vicinity of a multivesicular body. Scale-bar: $0.5 \mu \mathrm{m}$

Fig 3. Section half way along the regenerated portion of a barbel, 5 days after operation. Collagen fibrils are enclosed in lysosomes (arrows) in a fibroblast. Scale-bar: $0.5 \mu \mathrm{m}$

Fig 4. Similar to Fig. 3, showing a fibroblast with rER, part of a Golgi system, lysosomes, and enclosed collagen fibrils (arrows) the identity of which had been established at greater magnification on the screen. Scale-bar: $0.5 \mu \mathrm{m}$.

$c$ Extracellular collagen fibrils, $f$ fibroblast, $g$ Golgi system, $n$ nucleus 
istic cytoplasm, with scattered free ribosomes and usually with extensive sacs of ribosomal endoplasmic reticulum. Many of the fibroblasts contained numerous membrane-enclosed fibrils, which in transverse section had diameters matching those of the collagen fibrils of the matrix (Fig. 1). The membrane usually enclosed one or two fibrils, occasionally more. These fibroblasts also contained multivesicular bodies and profiles with electron-dense granular contents, identified as lysosomes on grounds of fine structure. A few of the lysosomal profiles contained myelin bodies, but many contained fibrils similar to the collagen fibrils, together with granular material and occasionally vesicles as in Figure 2, where there is also a membranous extension from one pair of enclosed fibrils to the vicinity of a multivesicular body.

Within the regenerated portions of the barbels, there was no skeletal rod. The axis contained blood capillaries, regenerating nerves with Schwann cells, and a mass of fibroblasts, interspersed with collagen of fibril diameter $20-25 \mathrm{~nm}$, which was not orientated as regularly as that proximal to the wound site. Figure 3 and 4 are from sections at a level where the collagen had been formed, probably, two or three days before. Fibroblasts in the axis contained lysosomal profiles and multivesicular bodies, as at the wound site, and membrane-bounded collagen fibrils, with diameters matching those in the matrix. Fibrils in the lysosomes also resembled those of the matrix (Fig. 3). In the regenerated connective tissue, individual fibroblasts contained cisternae of ribosomal endoplasmic reticulum and well developed Golgi systems, sometimes with vesicles of dense fibrous contents, distinct from the membrane-enclosed fibrils, although these structures might be in close proximity (Fig. 4). Near the growing tip of the barbel, where less extracellular collagen was present, membrane-enclosed collagen fibrils were also seen in fibroblasts, three, five and seven days after operation.

\section{DISCUSSION}

In situations of rapid turnover of connective tissue, as in a healing wound, the presence of recognizable collagen fibrils enclosed by membranes, within fibroblasts, can be taken to indicate that phagocytosis and degradation play a part in connective tissue remodelling (TEN CATE and FreEman, 1974). Svoboda et al. (1981) found a clear positive correlation between such internal profiles and the rate of collagen turnover in particular tissues. The images from the fish barbel match those reported from mammalian tissues.
MARCHI and LEBLOND (1983) summarise the longstanding controversy on whether collagen fibrils are assembled inside or outside the cell, and support the extracellular mechanism (see also LEBLOND, 1989). They found that a small proportion of labelled collagen precursors might deviate towards the lysosomal pathway but that the bulk was added to extracellular fibrils; no new material was incorporated into the collagen fibrils that were enclosed by membrane inside the cell. MELCHER and CHAN (1981) used serial sections to confirm that many fibrils were indeed completely enclosed within the cell and that there was evidence of digestion within such spaces as well as in more typical lysosomal profiles. In the present instance, which involves wound-healing followed by rapid regeneration of the barbel, recentlyformed collagen fibrils could be distinguished from collagen existing before the experiment by their diameters, the new fibrils being thinner. This agrees with an observation of ASUWA (1988) in skin wounds of rabbit, that new fibrils are slender. In the catfish, there can be no doubt that the enclosed fibrils at the level where the barbel was cut were old collagen. Identification of lysosomes was dependent on fine structural features, because it was not practicable to visualise the enzymes at the time, but images that could represent intermediate stages between multivesicular bodies and lysosmal profiles containing fibrils similar to those enclosed elsewhere in the cell, were seen in numerous instances, both at the level of the wound and in the regenerated tissue. It is a reasonable interpretation that collagen fibrils were in process of ingestion and digestion at both sites. The presence of enclosed fibrils in recently-formed tissue suggests that remodelling involves recycling of fibrils of collagen. Such adjustment at the tissue level is distinct from the destruction of new collagen at the cellular level, which BIENKOWSKI et al. (1978) and BERG and NEBLOCK (1985) demonstrated but considered too rapid to involve phagocytosis. The number of lysosomes present is compatible with both activities, and of course some lysosomes could be involved in other cellular cycles.

The hypothesis, of phagocytosis of newly-formed extracellular fibrils, provides for the rearrangements necessary in a growing regenerate, and such activity would ensure a ready supply of the appropriate materials for resynthesis. It is not necessary to assume, as did HENELL et al. (1983) and MichnA (1988), that these enclosed fibrils were being degraded before they had left the cell. The hypothesis also provides an alternative explanation of the enclosed profiles seen in embryonic tendon and cornea, which 
TRELSTAD and HAYASHI (1979) and BIRK and TREL. STAD (1984) interpreted as evidence of the formation of fibrils in deep pits of the fibroblast surface. YAJIMA (1988) claimed that secretion and phagocytosis of collagen occur in separate phases of activity, but his material was cultured and extraneous collagen was involved. The presence of rER, secretory bodies from the Golgi, lysosomes and membrane-enclosed fibrils, in individual fibroblasts of the growing regenerated barbel, suggests that synthesis and breakdown can occur simultaneously. Enclosed collagen fibrils were not reported by ZYLBERBERG et al. (1988) in hyposquama of regenerating teleost scales, but that is a special situation where the orientation of the collagen fibrils is strictly controlled at the time of formation and the cells retreat from the scale as it is formed. In a catfish barbel, the fibrils of the growing regenerate do not have a strict longitudinal orientation and remodelling of the connective tissue during the regeneration process is implied, if the structure is to re-acquire its normal morphology.

\section{REFERENCES}

Asuwa, N.: Collagen degradation in the rabbit skin during short-term tissue culture. Virchows Arch. B, Cell. Pathol. 55: 345-354 (1988).

BAECKer, R.: Beitrage zur Histologie der Barteln der Fische. Z. Mikrosk.- Anat. Forsch. 6: 489-507 (1926).

BERG, R. A. and D. S. NeBlock: Intracellular degradation of newly synthesized collagen. Ann. New York Acad. Sci. 460: 395-396 (1985).

Bienkowski, R. S., M. J. Cowan, J. A. McDonald and R. G. CRYstal: Degradation of newly synthesized collagen. J. Biol. Chem. 253: 4356-4363 (1978).

Birk, D. E. and R. L. Trelstad: Extracellular compartments in matrix morphogenesis: collagen fibril, bundle and lamellar formation by corneal fibroblasts. J. Cell Biol. 99: 2024-2033 (1984).

Henell, F., J. L. E. Ericsson and H. Glaumann: An electron microscopic study of the post-partum involution of the rat uterus. Virchows Arch. B, Cell. Pathol. 42: 271-287 (1983).
LEblond, C. P.: Synthesis and secretion of collagen by cells of connective tissue, bone and dentin. Anat. Rec. 224: 123-138 (1989).

Marchi, F. and C. P. Leblond: Collagen biogenesis and assembly into fibrils as shown by ultrastructural and $3 \mathrm{H}$-proline radioautographic studies on the fibroblasts of the rat foot pad. Amer. J. Anat. 168: 167-197 (1983).

Melcher, A. H. and J. Chan : Phagocytosis and digestion of collagen by gingival fibroblasts in vivo: a study of serial sections. J. Ultrastr. Res. 77:. 1-36 (1981).

MichNA, H.: Intracellular collagen fibrils: evidence of an intracellular source from experiments with tendon fibroblasts and fibroblastic tumour cells. J. Anat. 158: 1-12 (1988).

Svoboda, E. L. A., A. Shiga and D. A. Deporter: A stereologic analysis of collagen phagocytosis by fibroblasts in three soft connective tissues with differing rates of collagen turnover. Anat. Rec. 199: 473-486 (1981).

Ten Cate, A. R. and E. Freeman: Collagen remodelling by fibroblasts in wound repair. Preliminary observations. Anat. Rec. 179: 543-546 (1974).

Trelstad, R. L. and K. HaYashi: Tendon collagen fibrillogenesis: intracellular subassemblies and cell surface changes associated with fibril growth. Devel. Biol. 71: 228-242 (1979).

Y AJIMA, T.: Localization of acid phosphatase activity in collagen-secreting and collagen-resorbing fibroblasts. Histochemistry 90: 245-254 (1988).

Zylberberg, L., J. Bereiter-Hahn and J.-Y. Sire: Cytoskeletal organization and collagen orientation in the fish scales. Cell Tiss. Res. 253: 597-607 (1988).
Dr. Mary WhITEAR

10, Parkwood Road

Tavistock

Devon PL19 0HH

United Kingdom 